\title{
773 A NOVEL CANCER IMMUNOTHERAPY; VACCINATION AGAINST TUMOR VASCULAR EXTRACELLULAR VIMENTIN
}

Elisabeth Huijbers*, Judy van Beijnum, Karlijn van Loon, Arjan Griffioen. Amsterdam UMC, Cancer Center Amsterdam, Amsterdam, Netherlands

Background Angiogenesis, the development of neovasculature, is required to sustain tumor growth and metastasis of solid tumors. The tumor neovasculature expresses specific markers, which are selectively overexpressed in tumor endothelial cells compared to normal healthy adult endothelium and are therefore ideal targets for vaccination.

Methods One of these tumor vascular markers is the extracellular cytoskeletal protein vimentin. This marker is externalized from tumor endothelial cells, while expression in all other cells in the body is exclusively intracellular. Extracellular vimentin (eVim) is pro-angiogenic and functionally mimics vascular endothelial growth factor (VEGF) action, while concomitantly acting as inhibitor of leukocyte-endothelial interactions, thereby hampering leukocyte infiltration into the tumor. eVim is overexpressed in the vasculature of different solid tumors, but not present in normal healthy tissue.

Results Using iBoost, our proprietary conjugate vaccine technology for induction of efficient antibody responses against self-antigens, we were able to generate strong eVim specific humoral immune responses, resulting in inhibition of tumor growth in preclinical models without affecting the normal healthy vasculature. Furthermore, in an ongoing clinical study in client owned dogs with spontaneous bladder cancer our eVim vaccine shows effective and safe inhibition of angiogenesis and tumor growth.

Conclusions Targeting of extracellular vimentin by vaccination therefore presents a promising antiangiogenic immunotherapy strategy that is currently translated into clinical testing.

http://dx.doi.org/10.1136/jitc-2021-SITC2021.773 